Commun:

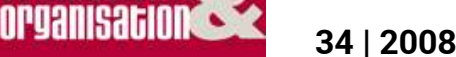

Retour sur les images d'organisations

\title{
Représentations sociales et iconographie
}

Pascal Moliner

\section{OpenEdition}

Journals

Édition électronique

URL : http://journals.openedition.org/communicationorganisation/547

DOI : 10.4000/communicationorganisation.547

ISSN : 1775-3546

Éditeur

Presses universitaires de Bordeaux

Édition imprimée

Date de publication : 1 décembre 2008

Pagination : $12-23$

ISBN : 978-2-86781-582-9

ISSN : 1168-5549

Référence électronique

Pascal Moliner, «Représentations sociales et iconographie », Communication et organisation [En ligne], 34 | 2008, mis en ligne le 01 décembre 2011, consulté le 19 avril 2019. URL : http:// journals.openedition.org/communicationorganisation/547; DOI : 10.4000/ communicationorganisation.547 
Dossier : Retour sur les images d'organisations

\title{
Résumé
}

A partir des réflexions de Moscovici concernant le caractère figuratif des représentations sociales, on se penche sur les liens entre iconographie et représentation. L'examen de quelques propriétés des images visuelles, des images mentales et de leur rapport au langage conduit à s'intéresser au discours mythique comme illustration des associations entre figure et signification. Cette analyse est transférée au cas de la représentation sociale du monde de l'Entreprise, abordée sous l'angle de l'approche structurale. Une étude expérimentale montre que les éléments de cette représentation peuvent générer de l'imagerie mentale et que c'est parmi les éléments centraux que l'on rencontre ceux qui génèrent l'imagerie la plus consensuelle. On s'interroge enfin sur les propriétés de l'iconographie lorsqu'elle s'intègre dans des stratégies de diffusion, de propagation ou de propagande.

\section{Mots-clés}

Iconographie, images mentale, noyau, représentations sociales.

\begin{abstract}
From Moscovici's propositions concerning the figurative character of social representations, one considers the links between iconography and representation. The examination of some properties of the visual images, the mental images and their report to the language led to be interested in the mythical speech like illustration of associations between figure and significance. This analysis is transferred to the case of the social representation of the Firm, considered under the structural approach. An experimental study shows that the elements of this representation can generate mental imagery and that it is among the central elements that one meets those which generate the most consensual imagery. One wonders finally on the properties of the iconography when it is integrated in strategies of diffusion, propagation or propaganda.
\end{abstract}

\section{Key-Word}

Central core, iconography, mental images, social representations

Pascal Moliner est professeur de psychologie sociale à l'Université Paul Valéry - Montpellier III. EA 4210. 


\section{Représentations sociales et iconographie} Pascal Moliner

pascal.moliner@univ-montp3.fr

Dans sa théorie des représentations sociales (Moscovici, 1961) postule l'existence de liens étroits entre les images et les croyances collectives. Il écrit : «Dans le réel, la structure de chaque représentation nous apparaît dédoublée, elle a deux faces aussi peu dissociables que le sont le recto et le verso d'une feuille de papier : la face figurative et la face symbolique. Nous écrivons que: Représentation = Figure/signification, entendant par là qu'elle fait correspondre à toute figure un sens et à tout sens une figure. » (Moscovici, 1961, p.63). Dans cette proposition, le terme de figure doit se comprendre au sens d'une image perceptible. Selon Moscovici, cette structure binaire résulte des deux processus fondamentaux qui président à l'élaboration de toute représentation. Ces deux processus ont pour fonction « de doubler un sens par une figure, donc objectiver d'un côté... et une figure par un sens, donc ancrer de l'autre côté... les matériaux entrant dans la composition d'une représentation déterminée » (Moscovici, $1961: 64$ ). C'est le processus d'objectivation qui produit la figure et c'est le processus d'ancrage qui lui donne sens.

En décrivant la représentation comme l'association d'une figure et d'un sens, Moscovici propose une conception des représentations comme des processus symboliques. Mais cette conception ne se place pas, comme c'est le cas pour l'objectivation et l'ancrage, à un niveau génétique. Elle se situe au niveau du fonctionnement même des représentations sociales. Plus précisément, il s'agit de la description d'un certain mode de pensée que l'on pourrait aisément rapprocher de la pensée mythique. Car, ainsi que le note Lévi-Strauss (1962 : 32) : «les éléments de la réflexion mythique se situent toujours à michemin entre des percepts et des concepts ». Or, c'est bien cet alliage que l'on retrouve dans l'association figure/sens. Et c'est lui qui permet à certains éléments de devenir les signes de l'objet de représentation. $\mathrm{Au}$ sens où, comme l'entend de Saussure (1955), le signe est l'association d'une forme et d'un concept. Si, comme l'explique Moscovici (1961 : 62) : «L'inconscient est, dans l'esprit de la plupart d'entre nous, un signe de la psychanalyse », c'est parce que ce terme associe à l'image d'une couche profonde du cerveau, les notions de caché et d'involontaire. De cette association se dégage une signification nouvelle qui est directement rattachée à l'objet de représentation. C'est en ce sens qu'à notre avis, l'association 


\section{Dossier : Retour sur les images d'organisations}

figure/sens rend compte d'un processus ou d'un système particulier de signification.

Dans ce système, l'iconographie et l'imagerie mentale jouent un rôle prépondérant dans leurs liens avec le langage, porteur de significations. Cette problématique a déjà fait l'objet de diverses réflexions. Ainsi, pour De Rosa et Farr (2001), l'iconographie doit être à la fois considérée comme source, médium et produit des représentations sociales. Dans une autre direction, on a proposé de considérer les représentations comme des grilles de lecture permettant d'interpréter les images du monde et produisant en retour des « images sociales » de ce monde (Moliner, 1996, Brissaud, 2005). Enfin, pour les spécialistes des Sciences de l'Information et de la communication, la communication médiatique peut être envisagée comme une interaction sociale «production-dispositif-réception » entre des sujets sociaux dotés d'intentionnalité (Courbet, Fourquet-Courbet et Chabrol, 2006). Dans cette perspective, on peut alors penser que les représentations sociales et leurs contenus imagés participent du dispositif qui relie la production à la réception de l'iconographie.

Mais jusqu'à présent, ces différentes approches ont négligé le caractère structural des représentations sociales. En effet, autant pour Moscovici (1961), que pour Abric (1976), ces dernières s'organisent autour d'un noyau, figuratif pour le premier et signifiant pour le second. C'est donc probablement au sein de ce noyau que se réalise l'association figure / sens évoquée plus haut. L'objectif du travail que nous présentons ici est de fournir des arguments empiriques à cette thèse.

\section{La question de l'image.}

Quelle est l'image de la femme, ou des femmes, dans la publicité ? Voilà le genre de question un peu galvaudée qui pourrait constituer le point de départ d'un travail universitaire de troisième ou de quatrième année en psychologie ou dans d'autres disciplines des sciences sociales. Derrière cette question, trop simple en apparence, se cachent en réalité trois interrogations.

La première, la plus triviale, renvoie à l'iconographie et attire notre attention sur ce qui est montré des femmes dans la communication publicitaire. Pour y répondre, il conviendrait de dresser l'inventaire des différentes formes de cette iconographie.

La seconde, plus complexe, contient le pré supposé implicite que les diffuseurs de cette iconographie ont des croyances et des motivations et que pour y répondre, ils mettent en scène des images. Se pose alors 
la question de comprendre les liens entre les formes de l'iconographie et les croyances et intentions qui ont motivé son élaboration.

La troisième interrogation soulevée par notre question initiale est tout aussi complexe que la précédente. Si l'élaboration iconographique répond à des croyances et à des motivations, elle doit s'inscrire dans un processus de communication. Elle doit donc respecter un code commun aux émetteurs et aux récepteurs des images. Quels sont alors les éléments de ce code?

$\mathrm{Au}$ fond, notre question initiale porte à la fois sur des matériaux iconographiques dont les méthodes d'analyse restent à inventer et sur des systèmes de croyances partagées qui permettent aux individus de communiquer par le biais d'images. Lorsque ces communications concernent des objets sociaux, ces systèmes sont des représentations sociales qui orientent tout autant l'élaboration des formes iconographiques que leur interprétation. Le problème ainsi posé renvoie à trois niveaux d'analyse : perceptif, cognitif et symbolique.

$\mathrm{Au}$ niveau perceptif, le support iconographique, représentation graphique de «quelque chose » présente trois propriétés essentielles. D'abord son pouvoir de figuration, c'est-à-dire sa capacité à se substituer aux objets qu'il montre. Ensuite sa «capacité d'éveil » (Gombrich, 1983), c'est-à-dire sa capacité à générer des émotions. Enfin son ambiguïté qui oblige le spectateur à faire des choix et, par là même, à s'approprier le sens de l'image qu'il perçoit.

Au niveau cognitif, l'image devient «mentale». Il s'agit de l'évocation mentale d'une expérience perceptive antérieure (Denis, 1989) et il est possible de réaliser à partir d'elle, les mêmes opérations que celles autorisées par une perception directe. Mais l'imagerie mentale n'est pas qu'une simple trace de la perception visuelle. Piaget et Inhelder (1966), remarquent en effet, que les manifestations de l'imagerie mentale ne sont observées chez l'enfant qu'à partir de l'apparition de la fonction sémiotique. En d'autres termes, la fonction d'imagerie mentale repose sur la capacité à manipuler des signes, c'est-à-dire à associer une forme et un sens.

Dans les systèmes de signes, les formes, on le sait peuvent être diverses. Elles sont les «signifiants »; les vecteurs qui portent une signification que l'on appelle le «signifié» (Barthes, 1957; de Saussure, 1955). Dans le cas de l'imagerie mentale, les signes dont il est question associent des éléments figuratifs à des éléments sémantiques, signifiés par des mots. On retrouve, alors, l'association 


\section{Dossier : Retour sur les images d'organisations}

figure / sens évoquée plus haut. Mais parce qu'il s'agit justement d'un système sémiotique, chacun de ces termes envoie à l'autre.

Au niveau symbolique, l'image est un processus. A la capacité que nous avons de faire correspondre un mot à une image, répond la capacité de certains mots à susciter en nous des images. La psychologie cognitive parle, en effet, de la «valeur d'imagerie » d'un mot comme étant «la capacité qu'a ce mot de susciter une image mentale chez l'individu» (Denis, 1989, p.138). En d'autres termes, l'association d'une image mentale à une signification réalise un processus symbolique.

Le récit mythique nous semble être une parfaite illustration de ce processus où s'associent des images et des significations. Il est facile, en effet, d'y repérer des éléments figuratifs et des éléments informatifs. La combinaison de ces éléments dans les représentations picturales du mythe permet d'orienter son interprétation dans des directions diverses ${ }^{1}$. En d'autres termes, la communication qui s'établit entre les peintres et les spectateurs n'est possible que dans la mesure où les uns et les autres partagent une connaissance commune. Elle permet aux premiers de construire leurs images en leur fournissant des éléments iconographiques et des éléments de scénario. Elle permet aux seconds d'interpréter ces images et de leur donner un sens à travers la reconnaissance des éléments qu'elles contiennent. C'est là, selon nous, l'illustration du rôle que peut jouer un savoir partagé dans l'élaboration et l'interprétation des images. Ajoutons ici que l'on trouve la trace de ce phénomène dans une étude récente qui montre comment des concepteurs publicitaires mobilisent des représentations sociales dans le processus de création d'images (Courbet et FourquetCourbet, 2005).

\section{Représentation sociale et imagerie mentale du monde de l'entreprise ${ }^{2}$.}

Rappelons tout d'abord que selon l'approche structurale des représentations (Abric, 1976, 1994), ces dernières seraient organisées selon un double système. Le système «central», constitué de cognitions relativement abstraites (Moliner, 1988), et consensuelles, aurait pour fonction première de déterminer la signification globale

1 Songeons par exemple à quelques représentations picturales du mythe d'Icare : Saraceni, 1575-1620 ; Lebrun, 1645 ; Blondel, 1819.

2 Les données présentées dans cette recherche ont été recueillies par G. Hirailles dans le cadre d'un mémoire de Master. 
d'une représentation. Le système «périphérique » serait, quant à lui, constitué d'éléments beaucoup plus concrets, renvoyant aux expériences et aux pratiques individuelles relatives à l'objet. On dispose aujourd'hui de nombreux travaux confortant la validité de cette approche théorique (Moliner, 1989, 1993, Rateau, 1995, Abric et Tafani, 1995). Par ailleurs, on a pu montrer que les éléments centraux se distinguaient par leur fort pouvoir associatif. La méthode des Schèmes Cognitif de Base montre, en effet, que les liens associatifs entre des éléments centraux et d'autres éléments d'une représentation sont nombreux et variés (Guimelli et Rouquette, 1992). Enfin, des travaux plus récents suggèrent que les cognitions centrales seraient davantage polysémiques que les cognitions périphériques (Moliner et Martos, 2005). En d'autres termes, en raison de leur polysémie, les éléments centraux auraient la propriété d'évoquer de nombreuses significations.

A partir de ces considérations, si l'on suppose que les représentations sociales jouent, dans l'élaboration et l'interprétation des images, un rôle comparable à celui des récits mythiques, on doit faire l'hypothèse que comme eux, elles réalisent des associations figure / signification. Cela signifie donc que parmi les éléments qui constituent une représentation, on devrait rencontrer, comme dans les discours mythiques, des éléments à forte capacité d'imagerie mentale. C'est pour tester cette hypothèse que nous avons réalisé l'expérience qui suit.

\section{Matériel, procédure et hypothèses.}

Pour cette expérience, nous avons utilisé un matériel lexical extrait des recherches sur la représentation du monde de l'entreprise chez des étudiants (Moliner, 1993, 1996). Nous avons ainsi sélectionné trois mots désignant des éléments périphériques de cette représentation (emploi, organisation et production), et quatre mots désignant des éléments centraux (concurrence, économie, patron et profit). La procédure consistait à présenter ces mots à des sujets comparables à ceux des études antérieures. On indiquait que l'expérience concernait le « monde de l'entreprise », et on leur lisait chacun des 7 mots. Après l'audition de chaque mot, on demandait aux participants d'indiquer l'image mentale qui leur venait à l'esprit ${ }^{3}$. Les 30 participants à l'expérience étaient interrogés individuellement.

3 En dépit de sa simplicité, ce type de consigne est relativement fréquent dans les recherches de psychologie cognitive (Bonin et al., 2003). 


\section{Dossier : Retour sur les images d'organisations}

Puisque les éléments périphériques correspondent à des expériences individuelles, on s'attendait à ce que l'imagerie qu'ils génèrent soit disparate d'un individu à l'autre. Au contraire, on s'attendait à ce que les éléments centraux génèrent une imagerie mentale plus consensuelle.

D'autre part, puisque ce sont les éléments centraux qui structurent les représentations, on s'attendait à rencontrer des éléments centraux figuratifs, à forte capacité d'imagerie et des éléments centraux informatifs, à faible capacité d'imagerie mentale.

\section{Résultats}

Les 210 réponses obtenues ont fait l'objet d'une analyse de contenu faisant apparaître sept catégories. Le tableau 1 présente, pour chaque catégorie, des exemples de réponses ainsi que le nombre de réponses obtenues. Notre analyse fait apparaître cinq types d'images mentales et une catégorie de réponses ne correspondant pas à des images mentales. Dans cette catégorie «autre» (22\% des réponses), nous avons regroupé les réponses qui n'indiquaient pas explicitement une image concrète (ex : système, relations publiques, hiérarchie), ainsi que les non réponses.

$\mathrm{Au}$ total 163 images mentales ont été identifiées. La première catégorie d'images regroupe des images d'objets ou de lieux (45\% du total des images recueillies), la seconde regroupe des images de personnes $(18 \%)$, la troisième rassemble des images de lieux de travail (15\%), la quatrième correspond à des dessins ou des représentations graphiques $(12 \%)$. Enfin la dernière catégorie d'images regroupe des situations, c'est-à-dire des images de personnes en action $(11 \%)$.

\begin{tabular}{|l|l|l|}
\hline Catégories & Exemples de réponses & $\mathrm{N}$ \\
\hline Objets ou lieux & Argent, produits, bourse & 72 \\
\hline Personnes & Un homme, un groupe, des salariés... & 30 \\
\hline Lieux de travail & Usine, entreprise, bureaux... & 24 \\
\hline Dessins & $\begin{array}{l}\text { Organigramme, emploi du temps, } \\
\text { graphique... }\end{array}$ & 19 \\
\hline Situations & Combat, réunion, course... & 18 \\
\hline Autre & Hiérarchie, Bac, Medef, non réponse. & 47 \\
\hline
\end{tabular}

Tableau 1. Catégorisation des réponses produites à partir des 7 inducteurs relatifs à la représentation du monde de l'entreprise. 
Cette première analyse montre que l'imagerie mentale suscitée par le matériel lexical utilisé renvoie massivement à des objets ou à des lieux. Mais il convient d'examiner comment se répartit cette imagerie en fonction des différents items. L'examen du tableau 2 montre que l'imagerie mentale suscitée par l'item « profit » se situe principalement dans la catégorie «objets ou lieux». Sur les trente participants interrogés, $19(63 \%)$ indiquent l'image de l'argent, sous forme de billets ou de pièces. L'imagerie suscitée par l'item «patron » se situe dans la catégorie «personnes». $43 \%$ des participants indiquent l'image d'un homme. Pour l'item " économie », c'est à nouveau l'image de l'argent qui est indiquée (26\%). Pour l'item « concurrence », ce sont des images de situations qui sont indiquées (16\%), notamment des situations compétitives (course, combat...). L'item «production» suscite des images mentales de lieux de travail avec comme réponse dominante l'image d'une usine $(23 \%)$. L'item « organisation » suscite l'image d'un dessin, soit un organigramme (10\%), soit un emploi du temps $(10 \%)$. Enfin l'item «emploi » évoque des images très variées qui, bien que regroupées au sein des catégories, renvoient à des objets, des lieux ou des personnes différentes.

\begin{tabular}{|l|c|c|c|c|c|c|c|c|}
\hline & $\begin{array}{c}\text { Objets } \\
\text { ou } \\
\text { lieux }\end{array}$ & Personnes & $\begin{array}{c}\text { Lieux } \\
\text { Te }\end{array}$ & & & & & $\begin{array}{c}\text { Rapport } \\
\text { T/O }\end{array}$ \\
\hline Profi & 22 & 2 & 1 & 3 & & 2 & 30 & $28 \%$ \\
\hline PATRON & 6 & 14 & 1 & & 2 & 7 & 30 & $47 \%$ \\
\hline ECONOMIE & 17 & 1 & 2 & 1 & & 9 & 30 & $52 \%$ \\
\hline CONCURRENCE & 6 & 5 & 4 & & 8 & 7 & 30 & $69 \%$ \\
\hline PRODUCTION & 9 & & 10 & & 3 & 8 & 30 & $59 \%$ \\
\hline ORGANISATION & 6 & 3 & 4 & 10 & 2 & 5 & 30 & $64 \%$ \\
\hline EMPLOI & 6 & 5 & 2 & 5 & 3 & 9 & 30 & $85 \%$ \\
\hline
\end{tabular}

Tableau 2. Répartition des réponses en fonction de l'inducteur et de la catégorie d'images mentales.

La dernière colonne du tableau 2 présente le rapport Type/Occurrence pour chaque item. Il s'agit du nombre d'images mentales différentes produites à partir d'un item, divisé par le nombre total d'images mentales produites à partir du même item. Une valeur faible de ce rapport indique une forte homogénéité des réponses. On constate alors que c'est l'item central «profit » qui donne lieu à l'imagerie la plus consensuelle $(\mathrm{T} / \mathrm{O}=28 \%)$, suivi par les items centraux «patron» 
$(\mathrm{T} / \mathrm{O}=47 \%)$ et «économie » $(\mathrm{T} / \mathrm{O}=52 \%)$. Il semble donc que ce soit parmi les éléments centraux que l'on rencontre les items donnant lieux à l'imagerie la plus consensuelle. Globalement, parmi les 95 images mentales suscitées par les éléments centraux, on dénombre 45 images différentes $(\mathrm{T} / \mathrm{O}=47 \%)$. En revanche, parmi les 68 images mentales suscitées par les éléments périphériques, on compte 47 images différentes $(\mathrm{T} / \mathrm{O}=69 \%)$. La comparaison de ces deux proportions indique une variabilité de l'imagerie périphérique supérieure à celle de l'imagerie centrale $(\mathrm{p}=.005)$, ce qui va dans le sens de notre première hypothèse.

Enfin, on peut s'intéresser à la capacité d'imagerie des éléments centraux. Pour ce faire, on a considéré que les réponses appartenant aux catégories «concepts» et «autre" » ne relevaient pas de l'imagerie mentale. Le tableau 3 présente donc, pour chaque item central, le nombre et la proportion d'images mentales suscitées (nombre d'images divisé par nombre total de réponses).

\begin{tabular}{|l|c|c|}
\hline & $\begin{array}{c}\text { Nombre d'images } \\
\text { mentales }\end{array}$ & $\begin{array}{c}\text { Proportion d'images } \\
\text { mentales }\end{array}$ \\
\hline PROFIT & 28 & $93 \%$ \\
\hline PATRON & 21 & $70 \%$ \\
\hline ECONOMIE & 23 & $76 \%$ \\
\hline CONCURRENCE & 23 & $76 \%$ \\
\hline
\end{tabular}

Tableau 3. Nombre et proportion d'images mentales suscitées par les items centraux.

On constate alors que l'item «profit» suscite significativement plus d'images mentales que l'item «patron » (Chi deux $=4.01, \mathrm{p}<.05)$, et tendanciellement plus d'images mentales que les items "économie" et « concurrence » (Chi deux=3.27, p=.07). Par contre on ne constate aucune différence entre les items «patron», «économie» et « concurrence ». Il apparaît donc que l'item «profit» est doté d'une valeur d'imagerie supérieure à celle des autres items centraux de la représentation du monde de l'entreprise. Ce résultat va, selon nous, dans le sens de notre seconde hypothèse.

$\mathrm{Au}$ terme de cette recherche, il apparaît que l'imagerie mentale associée à une représentation puisse s'envisager à la fois sous un angle quantitatif et qualitatif. On remarque, en effet (cf. tableau 2), que des items qui suscitent sensiblement la même quantité d'images mentales se distinguent quant au caractère consensuel de ces images. C'est par exemple le cas des items «profit» et «organisation». Mais on 
remarque aussi que des items qui suscitent des images relativement consensuelles, n'ont manifestement pas les mêmes valeurs d'imagerie sur le plan quantitatif. C'est le cas des items « profit» et «économie». Enfin, certains items génèrent une imagerie mentale relativement peu abondante et hétérogène. C'est typiquement le cas de l'item «emploi ». La question se pose alors de savoir d'où provient la diversité des cas de figure que nous observons. Est-elle une conséquence ordinaire du processus représentationnel? Est-elle spécifique de certains objets sociaux ? Est-elle le propre de certaines populations?

\section{Conclusion}

Nous venons de voir que si tous les éléments de la représentation sociale de l'Entreprise sont susceptibles de générer de l'imagerie mentale, c'est parmi les éléments centraux que l'on rencontre ceux qui génèrent l'imagerie la plus consensuelle. Ce qui suggère une équivalence entre les notions de «noyau figuratif» et de «noyau central ». Ces résultats doivent encore être regardés avec prudence car ils soulèvent de nombreuses questions méthodologiques et théoriques. Parmi celles ci, il en est une sur laquelle nous voudrions attirer l'attention en conclusion de ce travail. Elle concerne le rôle et la forme de l'iconographie dans les phénomènes de diffusion, de propagation et de propagande (Moscovici, 1961). De notre point de vue, lorsqu'une iconographie accompagne ces modes de communication, elle se doit de composer avec les représentations de l'audience et donc, avec l'imagerie mentale qui leur est associée. A partir de ce postulat, on peut supposer que l'iconographie de la diffusion sera riche et fera massivement appel aux éléments les plus consensuels de cette imagerie, parce que c'est une façon de conférer au propos un caractère à la fois concret et objectif. En revanche, l'iconographie de la propagation devrait être plus sobre et surtout plus sélective. En effet, puisque la propagation opère une sélection parmi les formes possibles de l'objet, elle ne devrait en montrer que certaines facettes. Enfin si la propagande ambitionne de réorganiser les représentations de l'audience, elle devrait introduire dans son iconographie des éléments étrangers à l'imagerie mentale générée par ces représentations. Pour valider ces hypothèses, il conviendrait bien sûr de connaître l'imagerie mentale associée à la représentation sociale d'un objet donné et de procéder à une analyse systématique des images de cet objet dans la communication médiatique. Nous croyons que ce type de recherche pourrait alors dessiner les contours d'une méthodologie nouvelle d'analyse de l'iconographie des objets sociaux. 
Dossier : Retour sur les images d'organisations

\section{Bibliographie}

ABRIC Jean Claude. Jeux, conflits et représentations sociales. Thèse de Doctorat d'Etat. Aix en Provence : Université de Provence, 1976.

ABRIC Jean Claude. «Les représentations sociales: aspects théoriques », in : Pratiques Sociales et Représentations. Paris : Presses Universitaires de France, 1994

ABRIC Jean Claude, TAFANI Eric. « Nature et fonctionnement du système central d'une représentation sociale: la représentation de l'Entreprise », in Cahiers Internationaux de Psychologie Sociale, 1995, 28, pp. 22-31.

BARTHES Roland. Mythologies. Paris : Seuil, 1957.

BRISSAUD Anne. Image, représentation sociale et catégorisation. Le cas des hommes politiques. Thèse de Doctorat en Psychologie Sociale. Montpellier : Université Paul Valéry, 2005.

BONIN Patrick; MEOT Alain; AUBERT Lydie, MALARDIER Nathalie, NIEDENTHAL Paula; CAPELLE TOCZEK MarieChristine. « Normes de concrétude, de valeur d'imagerie, de fréquence subjective et de valence émotionnelle pour 866 mots », in L'année Psychologique, 2003, 104, pp. 655-694.

COURBET Didier, FOURQUET-COURBET Marie-Pierre. Les processus psychologiques lors de la création publicitaire. Hermès, 2005, 41, pp. 67-74.

COURBET Didier; FOURQUET-COURBET Marie-Pierre, CHABROL Claude. "Sujets sociaux et medias : Débats et nouvelles perspectives en sciences de l'information et de la communication », in Questions de communication, 2006, 10, pp. 157-179.

DENIS Michel. Image et Cognition, Paris : PUF, 1989.

DE ROSA Annamaria S., FARR Robert. "Icon and symbol. Two sides of the coin in the investigation of social representations". In : BUSCHINI F., KALAMPALIKIS N. coord. Penser la vie, le social, la nature. Mélanges en hommage Serge Moscovici. Paris: Les Editions de la Maison des Sciences de l'Homme, 2001, pp. 237-256

DE SAUSSURE Ferdinand. Cours de Linguistique générale. Paris : Payot, 1955.

GOMBRICH Ernst H. L'écologie des images. Paris : Flammarion, 1983. 
GUIMELLI, C. ; ROUQUETTE M.L., «Contribution du modèle associatif des schèmes cognitifs de base à l'analyse structurale des représentations sociales, in Bulletin de Psychologie, 1992, 405, pp. 196-202.

JODELET Denise. «Représentations sociales : phénomènes, concept et théorie », in : MOSCOVICI S. Coord. Psychologie sociale. Paris : Presses Universitaires de France, 1984

LEVI-STRAUSS Claude., La pensée sauvage. Paris : Plon, 1962.

MOLINER Pascal, La représentation sociale comme grille de lecture. Thèse de doctorat en psychologie. Aix en Provence: Université de Provence, 1988.

MOLINER Pascal, "Validation expérimentale de l'hypothèse du noyau central des représentations sociales» in Bulletin de Psychologie, 1989, 42, pp. 759-762.

MOLINER Pascal. ISA : «L'Induction par Scénario Ambigu. Une méthode pour l'étude des représentations sociales», in Revue Internationale de Psychologie Sociale, 1993, 2, pp. 7-21.

MOLINER Pascal. "A two-dimensional model of social representations", in European Journal of Social Psychology, 1995, 1, pp. 27-40.

MOLINER Pascal, Images et représentations sociales. Grenoble : Presses Universitaires de Grenoble, 1996.

MOLINER Pascal, MARTOS A., «La fonction génératrice de sens du noyau des représentations sociales. Une remise en cause »? in; Papers On Social Representations, 2005, 14, pp. 1-9.

MOSCOVICI Serge, La psychanalyse, son image et son public. Paris: Presses Universitaires de France. 1961, (2 ${ }^{\mathrm{èm}}$ e édition, 1976).

PIAGET Jean., INHELDER Bernard, L'image mentale chez l'enfant. Paris : PUF, 1966.

RATEAU Patrick, «Le noyau central des représentations sociales comme système hiérarchisé. Une étude sur la représentation du groupe » in Les Cahiers Internationaux de Psychologie Sociale, 1995, 26, pp. 29-52. 